ORIGINAL ARTICLE

\title{
Presence of antidrug antibodies correlates inversely with the plasma tumor necrosis factor (TNF)- $\alpha$ level and the efficacy of TNF-inhibitor therapy in psoriasis
}

\author{
Róbert KUI, ${ }^{1}$ Brigitta GÁL, ${ }^{1}$ Magdolna GAÁL, ${ }^{1}$ Mária KISS, ${ }^{1}$ Lajos KEMÉNY, ${ }^{1,2}$ \\ Rolland GYULAI ${ }^{1,3}$ \\ ${ }^{1}$ Department of Dermatology and Allergology, University of Szeged, ${ }^{2}$ MTA-SZTE Dermatological Research Group, Szeged, \\ ${ }^{3}$ Department of Dermatology, Venereology and Oncodermatology, University of Pécs, Pécs, Hungary
}

\begin{abstract}
Antidrug antibodies have been shown to be associated with a loss of response during biologic therapy. Despite the potential association, there has been no report on the simultaneous monitoring of the following parameters in psoriasis: presence of neutralizing antibodies, plasma tumor necrosis factor (TNF)- $\alpha$ concentration, TNFi concentration and disease activity. Plasma concentrations of adalimumab, infliximab, etanercept and their respective antidrug antibodies, as well as plasma concentrations of TNF- $\alpha$ were measured in 77 psoriasis patients receiving biologic therapy, and the values were correlated with the clinical activity of the skin disease. Antidrug antibodies were identified in the plasma of $25 \%$ of infliximab-treated patients and $\mathbf{2 9 . 6} \%$ of adalimumab-treated patients, but not in the etanercept group. Clinical severity scores were significantly higher in the antibody-positive patients. In patients receiving infliximab or adalimumab therapy, the presence of antidrug antibodies was directly associated with reduced plasma TNF-inhibitor concentration and elevated plasma TNF- $\alpha$ level.
\end{abstract}

Key words: adalimumab, antidrug antibodies, etanercept, infliximab, psoriasis.

\section{INTRODUCTION}

Biologic therapies targeting tumor necrosis factor (TNF)- $\alpha$ are widely used in the treatment of psoriasis. To date, three TNF inhibitors (TNFi) are registered for the treatment of plaque psoriasis: infliximab, adalimumab and etanercept. Infliximab, a mouse-human chimeric monoclonal IgG1 antibody, and adalimumab, a fully human monoclonal immunoglobulin (Ig)G1 antibody, are specific for TNF. Etanercept is a fusion protein of human TNF receptor-2 and human IgG1 Fc. ${ }^{1}$

It has been shown that administration of TNFi may lead to the formation of antidrug antibodies (ADA) and the development of an immune response. ${ }^{2}$ The presence of ADA has been associated with decreased plasma drug level and a partial or complete loss of response in psoriasis patients. ${ }^{3,4}$ Antibodies against etanercept have no apparent effects on clinical response, ${ }^{5,6}$ whereas antibodies against infliximab or adalimumab have been associated with diminished clinical response. ${ }^{4,7}$

Despite the apparent link between the presence of ADA, the plasma TNFi concentration and the clinical activity of the skin symptoms, there has been no report as yet on the simultaneous monitoring of these parameters together with the plasma TNF- $\alpha$ concentration in psoriasis patients. Therefore, we performed a cross-sectional study to determine the potential correlation between the plasma levels of TNF- $\alpha$, the extent of antibody formation against adalimumab, infliximab and etanercept, and the plasma trough concentration of the TNFi in patients with plaque psoriasis.

\section{METHODS}

\section{Subjects}

This study was approved by the internal review board of the University of Szeged. Informed consent was obtained from all participants, and the study was conducted in full accordance with the principles of the Declaration of Helsinki and biologic therapeutic guidelines. Caucasian psoriasis patients treated for at least 12 weeks with a TNFi (infliximab, adalimumab or etanercept) at the Department of Dermatology and Allergology of the University of Szeged were enrolled in the study between October 2011 and January 2012. To be eligible for biologic treatment in Hungary, patients must fulfill the Hungarian national guidelines criteria: diagnosis of psoriasis vulgaris; severe psoriasis documented as Psoriasis Area and Severity Index (PASI) of more than 15, Dermatology Life Quality Index of more than 10 or affected body surface area of more than $10 \%$; lack of efficacy or intolerance to phototherapy or methotrexate, cyclosporin or acitretin treatments. Demographic and clinical data of the patients are presented in Table 1. PASI scores at

Correspondence: Róbert Kui, M.D., Department of Dermatology and Allergology, University of Szeged, 6 Korányi fasor, Szeged H-6720, Hungary. Email: kui.robert@med.u-szeged.hu

Received 15 September 2015; accepted 15 December 2015 
Table 1. Basic demographic and clinical data from the study population

\begin{tabular}{|c|c|c|c|c|}
\hline Treatment groups & Etanercept $(n=22)$ & Infliximab $(n=28)$ & Adalimumab $(n=27)$ & Total $(n=77)$ \\
\hline Age (years) $)^{\dagger}$ & $46.6 \pm 12.6(21-73)$ & $51.2 \pm 12.7(26-78)$ & $48 \pm 15.7(23-76)$ & $48.8 \pm 13.7(21-78)$ \\
\hline Male patients (\%) & $13(59.1)$ & $17(60.7)$ & $17(63)$ & $46(61.0)$ \\
\hline Bodyweight $(\mathrm{kg})^{\dagger}$ & $85.7 \pm 24.1(54-156)$ & $93.7 \pm 20.5(52-143)$ & $88.7 \pm 18.8(54-130)$ & $89.7 \pm 21.0(52-156)$ \\
\hline $\mathrm{BMI}^{\dagger}$ & $27.7 \pm 5(20.2-38.5)$ & $32.4 \pm 7.3(18.4-53.9)$ & $30.1 \pm 5.3(20.8-42.2)$ & $30.2 \pm 6.3(18.4-53.9)$ \\
\hline $\begin{array}{l}\text { Patients with a history } \\
\text { of smoking }(\%)\end{array}$ & $8(36.4)$ & $10(35.7)$ & $10(37)$ & $28(36.4)$ \\
\hline $\begin{array}{l}\text { Duration of current biologic } \\
\text { therapy (months) }\end{array}$ & $28.4 \pm 18.8(5-66)$ & $19.9 \pm 16.4(3-63)$ & $17.8 \pm 10.4(3-39)$ & $21.6 \pm 15.8(3-66)$ \\
\hline $\begin{array}{l}\text { Patients receiving a } \\
\text { different biologic } \\
\text { therapy previously (\%) }\end{array}$ & $8(36.4)$ & $8(28.6)$ & $20(74.1)$ & $36(46.7)$ \\
\hline \multirow{4}{*}{ Previous biologic therapies } & Infliximab: 3 & Etanercept: 2 & Etanercept: 4 & \\
\hline & Adalimumab: 2 & Adalimumab: 4 & Infliximab: 10 & \\
\hline & Efalizumab: 1 & Efalizumab: 1 & Etanercept, infliximab: 3 & \\
\hline & Ustekinumab: 2 & Adalimumab, etanercept: 1 & $\begin{array}{l}\text { Etanercept, efalizumab: } 2 \\
\text { Efalizumab: } 1\end{array}$ & \\
\hline $\begin{array}{l}\text { PASI score at induction of } \\
\text { first biologic therapy }\end{array}$ & $16.6 \pm 4.6(9.6-25.6)$ & $18.4 \pm 5.6(9.3-32.7)$ & $20.8 \pm 9.2(4.1-52.1)$ & $18.7 \pm 7.0(4.1-52.1)$ \\
\hline $\begin{array}{l}\text { PASI score at induction of } \\
\text { current biologic therapy }\end{array}$ & $14.7 \pm 5.7(1.2-25.6)$ & $17.9 \pm 5.5(9.3-32.7)$ & $15.0 \pm 5.0(5.8-26)$ & $16.0 \pm 5.5(1.2-32.7)$ \\
\hline $\begin{array}{l}\text { Patients with psoriatic } \\
\text { arthritis }(\%)\end{array}$ & 7 (31.8) & $13(46.4)$ & $14(51.9)$ & $34(44.2)$ \\
\hline $\begin{array}{l}\text { Patients receiving } \\
\text { concomitant } \\
\text { immunosuppressive } \\
\text { therapy (\%) }\end{array}$ & $0(0)$ & $8(28.6)$ & $1(3.7)$ & $9(11.7)$ \\
\hline
\end{tabular}

${ }^{\dagger}$ Mean \pm standard deviation. BMI, body mass index; PASI, Psoriasis Area and Severity Index.

the initiation of the patients' first and current biologic therapy were retrieved from the patients' records. PASI scores were also calculated at the time of study enrolment.

\section{Measuring plasma TNF- $\alpha$ and TNFi trough concentrations and detecting ADA}

Blood samples (5-10 $\mathrm{mL}$ ) were obtained from patients on the days of scheduled infusion/injection. To ensure that the trough or residual plasma drug concentration was measured, blood was drawn a few hours before the scheduled administration of the TNFi. Plasma aliquots separated from the blood samples were frozen at $-20^{\circ} \mathrm{C}$. The plasma concentrations of TNF- $\alpha$ and the biologic therapeutic agent (infliximab, adalimumab or etanercept), and the presence of IgG-type ADA were determined by using an enzyme-linked immunosorbent assay (ELISA), in accordance with the manufacturer's recommendations (Matriks Biotek Laboratories, Ankara, Turkey). For the detection of ADA (antibodies to infliximab/adalimumab/etanercept), indirect ELISA methodology was used. In these assays, infliximab/adalimumab/etanercept is coated on the wall of the microtiter wells. During the first incubation period, antidrug antibodies are captured by binding to the drugs. After washing away the unbound components from samples, a peroxidaselabeled ADA-specific conjugate is added to each well and then incubated. The bound enzymatic activity is detected by addition of tetramethylbenzidine (TMB) chromogen substrate. For detecting plasma infliximab/adalimumab/etanercept, solid phase ELISA, based on the sandwich principle, were used. Standards and samples are incubated in the microtiter plate coated with a monoclonal antibody or reactant specific for infliximab/adalimumab/etanercept. After incubation, the wells are washed. A horse radish peroxidase conjugated (in case of infliximab and adalimumab) or a biotin-labeled (in case of etanercept) tracer monoclonal antibody against the TNFi drug is added and binds to the TNFi captured by the first monoclonal antibody or reactant on the surface of the wells. Following incubation, wells are washed and then the bound enzymatic activity is detected by addition of chromogen substrate. The TNF- $\alpha$ determination kit was also based on double monoclonal antibody sandwich assay. TNF- $\alpha$ is captured by a monoclonal antibody to human TNF- $\alpha$ coated on the wall of the microtiter wells, and detected by a second, peroxidase-labeled monoclonal antibody. The bound enzymatic activity is detected by addition of TMB chromogen substrate. According to the manufacturer, these assays detect only free plasma TNF- $\alpha$, etanercept, infliximab, adalimumab or ADA levels, while TNFi-TNF- $\alpha$ or ADA-TNFi complexes are not measured.

\section{Statistical analysis}

The plasma concentrations of TNF- $\alpha$, the trough concentrations of the TNFi and the presence of ADA were correlated with the patients' epidemiological and clinical data. Data were evaluated with the MedCalc version 12.2.1.0 (MedCalc Software, Ostend, Belgium) and SPSS version 15.0 (SPSS, Chi- 
cago, IL, USA) statistical programs. ADA-positive and -negative groups were analyzed using mixed factorial ANOVA and logistic regression. For other differences between groups, the paired $t$ test, the $\chi^{2}$-test and Fisher's exact test were performed, where appropriate. $P<0.05$ was considered statistically significant.

\section{RESULTS}

\section{Patient characteristics}

Of the 77 patients, 22 (28.6\%), 28 (36.4\%) and 27 (35.1\%) received etanercept, infliximab and adalimumab treatment, respectively (Table 1). The maintenance dose was $5 \mathrm{mg} / \mathrm{kg}$ i.v. every 8 weeks for infliximab, $40 \mathrm{mg}$ s.c. every 2 weeks for adalimumab and $50 \mathrm{mg}$ s.c. every week for etanercept. The groups receiving the three different TNFi treatments did not differ significantly in the following demographic and clinical characteristics: mean age, sex ratio, mean bodyweight, mean body mass index, proportion of smokers, mean duration of current biologic therapy, mean PASI score at induction of first biologic therapy, mean PASI score at induction of current biologic therapy and presence of psoriatic arthritis. Before the current biologic therapy, 30 patients received one, and six patients received two biologic agents. The patients in the adalimumab group had previously been treated significantly more frequently with biologic therapy than those in the other treatment groups (adalimumab vs etanercept, $P<0.05$; adalimumab vs infliximab, $P<0.01$ ), while no statistically significant difference was observed between the etanercept and infliximab groups $(P=0.76)$ in this regard. Of the 77 patients, nine received concomitant disease-modifying antirheumatic drug (DMARD) therapy (eight methotrexate and one leflunomide). Concomitant DMARD therapy was more frequent in the infliximab group than in the other two treatment groups.

\section{ADA, plasma trough TNFi concentrations, plasma TNF- $\alpha$ levels and clinical response}

Antidrug antibodies were detected in 15 of the 77 patients (19.5\%): seven and eight in patients receiving infliximab and adalimumab, respectively (Table 2). ADA were not identified in any of the 22 patients receiving etanercept therapy. Interestingly, most of the ADA (11/15) were detected in patients who received the TNFi therapy for up to 12 months, although the number of patients in this group was comparable with the number of patients receiving treatment for more than 12 months (22 and 33, respectively; $P<0.01$ ).

The mean plasma trough concentrations (i.e. residual concentrations prior to the next scheduled treatment) of adalimumab, etanercept and infliximab were $2.4 \pm 2.3$ (range, 06.9), $7.6 \pm 2.0$ (range, 4.1-11.3) and $2.9 \pm 0.8 \mu \mathrm{g} / \mathrm{mL}$ (range, 2.2-5.0), respectively. The mean plasma trough concentration of infliximab in ADA-negative patients was significantly higher than in the ADA-positive patients $(3.0 \pm 0.8$ [range, 2.2-5.0] and $2.3 \pm 0.1 \mu \mathrm{g} / \mathrm{mL}$ [range, $2.2-2.5]$, respectively $[P<0.05]$ ]) (Fig. 1a). In ADA-negative patients, the residual plasma adalimumab concentration was $2.8 \pm 2.3 \mu \mathrm{g} / \mathrm{mL}$ (range, 0-6.9), whereas in ADA-positive patients it was $1.5 \pm 2.2 \mu \mathrm{g} / \mathrm{mL}$ (range, 0-5.3) $(P=0.18)$ (Fig. 1b). As discussed in the previous section, ADA were not detected in the etanercept group.

The mean plasma TNF- $\alpha$ concentration was significantly lower in patients with negative anti-infliximab antibody status, compared with ADA-positive patients (2.5 \pm 3.9 [range, 0-14.4] and $13.2 \pm 12.9 \mathrm{pg} / \mathrm{mL}$ [range, $0-34.8$ ], respectively; $P<0.05$ ). Similarly, anti-adalimumab antibody-negative patients demonstrated a lower mean TNF- $\alpha$ concentration than the ADA-positive group $(1.0 \pm 2.0$ and $3.6 \pm 6.3 \mathrm{pg} / \mathrm{mL}$, respectively; $P=0.16)$. Interestingly, although patients receiving etanercept were ADA-negative, their mean TNF- $\alpha$ level $(7.0 \pm 6.3 \mathrm{pg} / \mathrm{mL})$ was significantly higher than that of ADA-negative patients receiving either infliximab or adalimumab (infliximab, $P<0.01$; adalimumab, $P<0.001$ ) (Fig. 2).

There was no statistically significant difference in the mean PASI score at the initiation of the therapy between the ADA-positive and-negative patients (data not shown). At the time of sample collection, however, the mean PASI score of the ADA-negative and the ADA-positive infliximab groups was significantly different $(1.6 \pm 1.6$ vs $9.3 \pm 11.2, P<0.05$, after adjusting for age and sex). Similarly, at the time of sample collection, the average PASI score of the ADA-negative, adalimumab-treated patients was significantly lower $(2.4 \pm 3.5$ vs $12.5 \pm 8.6, P<0.01$, after adjusting for age and sex) (Fig. 3).

Patients who received their first biologic therapy during the study (bio-naive patients), and patients who previously had received biologic therapy (non-bio-naive patients) did not differ

Table 2. Duration of the actual treatment, mean TNF- $\alpha$ plasma level, mean plasma TNFi level and PASI scores of patients at the time of sampling

\begin{tabular}{lccccc}
\hline & $\begin{array}{l}\text { Etanercept } \\
(n=22)\end{array}$ & $\begin{array}{l}\text { Adalimumab } \\
(n=19)\end{array}$ & $\begin{array}{l}\text { Adalimumab }+ \\
(n=8)\end{array}$ & $\begin{array}{l}\text { Infliximab } \\
(n=21)\end{array}$ & $\begin{array}{l}\text { Infliximab }+ \\
(n=7)\end{array}$ \\
\hline No. of patients with $\leq 12$ months of TNFi treatment & 4 & 5 & 5 & 6 & 6 \\
No. of patients with $>12$ months of TNFi treatment & 18 & 14 & 3 & 15 & 1 \\
TNF- $\alpha$ plasma level $(\mathrm{pg} / \mathrm{mL})^{\dagger}$ & $7.0 \pm 6.3$ & $1.0 \pm 2.0$ & $3.6 \pm 6.3$ & $2.5 \pm 3.9$ & $13.2 \pm 12.9$ \\
Plasma trough level of the TNFi $(\mu \mathrm{g} / \mathrm{mL})^{\dagger}$ & $7.6 \pm 2.0$ & $2.8 \pm 2.3$ & $1.5 \pm 2.2$ & $3.0 \pm 0.8$ & $2.3 \pm 0.1$ \\
PASI score at sampling & $2.5 \pm 2.5$ & $2.4 \pm 3.5$ & $12.5 \pm 8.6$ & $1.6 \pm 1.6$ & $9.3 \pm 11.2$ \\
Change of PASI score from baseline $(\%)^{\dagger}$ & $83.8 \pm 13.4$ & $83.9 \pm 19.1$ & $34.0 \pm 35.0$ & $90.2 \pm 11.1$ & $53.1 \pm 32.6$ \\
\hline
\end{tabular}

${ }^{\dagger}$ Mean \pm standard deviation. $-/+$, patients without/with antidrug antibodies (ADA). PASI, Psoriasis Area and Severity Index; TNFi, tumor necrosis factor inhibitor. 

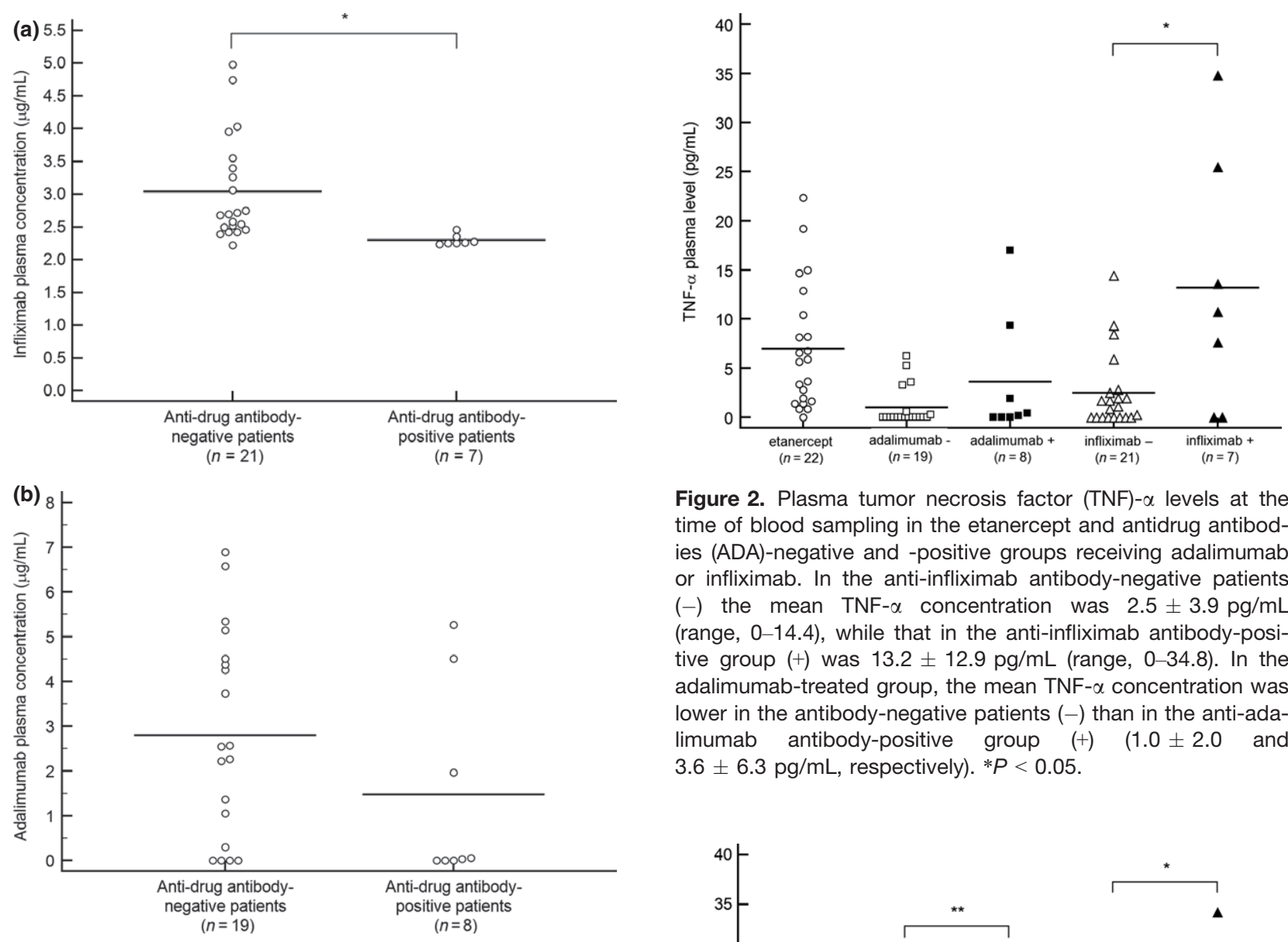

Figure 2. Plasma tumor necrosis factor (TNF)- $\alpha$ levels at the time of blood sampling in the etanercept and antidrug antibodies (ADA)-negative and -positive groups receiving adalimumab or infliximab. In the anti-infliximab antibody-negative patients $(-)$ the mean TNF- $\alpha$ concentration was $2.5 \pm 3.9 \mathrm{pg} / \mathrm{mL}$ (range, 0-14.4), while that in the anti-infliximab antibody-positive group (+) was $13.2 \pm 12.9 \mathrm{pg} / \mathrm{mL}$ (range, 0-34.8). In the adalimumab-treated group, the mean TNF- $\alpha$ concentration was lower in the antibody-negative patients $(-)$ than in the anti-adalimumab antibody-positive group $(+) \quad(1.0 \pm 2.0$ and $3.6 \pm 6.3 \mathrm{pg} / \mathrm{mL}$, respectively). ${ }^{*} P<0.05$.

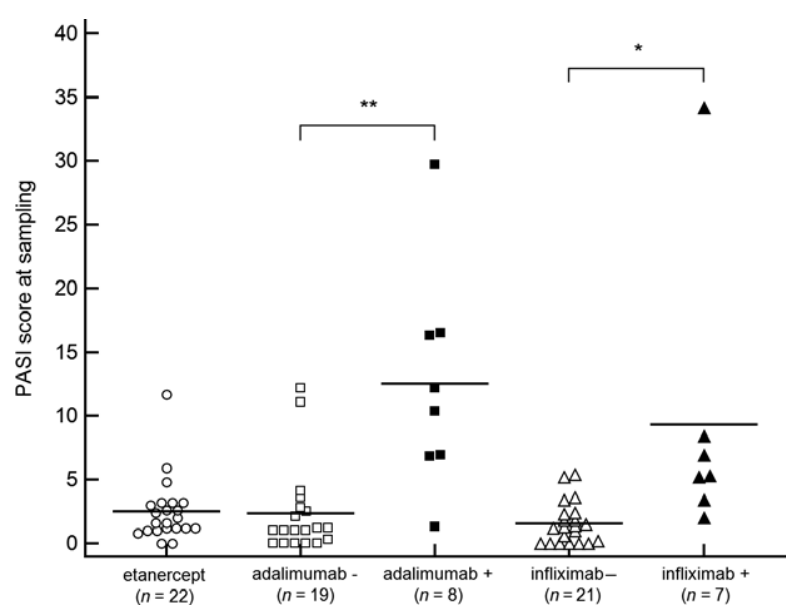

significantly in their clinical responses, extent of ADA-positivity and plasma TNF- $\alpha$ levels (data not shown).

\section{DISCUSSION}

The purpose of our cross-sectional study was to assess the presence of ADA against TNFi, the plasma trough concentrations of anti-TNF medications and the plasma TNF- $\alpha$ level during the biologic therapy of psoriasis, and their impact on the clinical efficacy.

Our results, that anti-infliximab or anti-adalimumab antibodies were detected in $25.0 \%$ and $28.6 \%$ of the respective patients, while ADA were not found against etanercept, are in agreement with previous reports. ${ }^{3-12}$ The clinical improvement

Figure 3. Psoriasis Area and Severity Index (PASI) scores at the time of blood sampling in the etanercept and antidrug antibodies (ADA)-negative and -positive adalimumab and infliximab groups. The mean PASI score at the time of blood sampling was significantly lower in the ADA-negative patients in both the infliximab and adalimumab groups (infliximab, $1.6 \pm 1.6$ vs $9.3 \pm 11.2$; adalimumab, $2.4 \pm 3.5$ vs $12.5 \pm 8.6$ in the ADAnegative vs -positive patients, respectively). ${ }^{*} P<0.05$, $* * P<0.01$.

among ADA-positive patients was significantly lower than in the ADA-negative groups. Previous studies similarly demonstrated significantly lower clinical efficacy of infliximab and 
adalimumab among ADA-positive patients, , ,7,8,12-14 as well as a clear correlation between decreased plasma infliximab level and poorer therapeutic outcome. ${ }^{13}$ Our observation that the plasma trough TNFi concentrations are lower in ADA-positive patients treated with infliximab and adalimumab fully supports these previous findings. The structure of the biologic agent is one of the most important factors determining the immunogenicity and the binding of ADA. Even though we have not detected neutralizing antibodies against etanercept, non-neutralizing ADA have been shown to form in etanercept patients. ${ }^{15}$ As these antibodies bind to the fusion region of etanercept, they leave the TNF-binding site free, and, thus, the function of etanercept remains uncompromised. However, as these immune complexes can cause quicker drug elimination, their formation may result in lower plasma trough levels and diminished therapeutic efficacy. ${ }^{2,16}$

Although the clinical activity of psoriasis was almost equal in the three treatment groups, the mean plasma TNF- $\alpha$ levels differed considerably. The lowest mean concentrations were measured in the adalimumab group and the highest in the etanercept treatment group. Interestingly, Kim et al. ${ }^{17}$ also describe this paradoxical phenomenon in a recent paper: they report that serum TNF- $\alpha$ concentrations in psoriasis patients increased after 12 weeks of etanercept treatment. In their opinion, serum TNF- $\alpha$ level after etanercept treatment reflected the summation of circulating TNF- $\alpha$-etanercept complexes with pretreatment free TNF- $\alpha$ levels. This could be a plausible explanation to our finding as well, however, the ELISA kits used in our experiments (according to the manufacturer) only detect free TNF- $\alpha$ (TNFi-TNF- $\alpha$ complexes are not measured). Obviously, either the ELISA kits after all detect both free and bound plasma TNF- $\alpha$, or there are other, currently unknown mechanisms which lead to increased TNF levels during etanercept treatment. As we did not find any direct correlation between clinical activity (PASI score) and the measured plasma TNF- $\alpha$ levels in our cohort of etanercept-treated patients, this latter explanation, however, seems more unlikely. The mean plasma TNF- $\alpha$ concentration was significantly higher among the ADA-positive infliximab- and adalimumab-treated patients than among the ADA-negative patients. Notably, this study has not established a clear statistical correlation between the plasma TNF- $\alpha$ level and the clinical activity of psoriasis during TNFi therapy. This result may be due in part to our relatively small study population, as patients who responded well tended to have lower TNF- $\alpha$ levels. It must also be considered, however, that the plasma TNF- $\alpha$ concentration is not necessarily associated directly with the efficacy of TNFi therapy. Other indicators (such as the TNF- $\alpha$ concentration in the lesional psoriatic skin) may correlate more closely with the clinical activity of psoriasis during anti-TNF treatment. A recent report clearly showed that TNF blockers decrease the activity of multiple pro-inflammatory pathways in lesional skin, including the activation and maturation of dendritic cells, the activation of $\mathrm{T}$ lymphocytes, and the production of different cytokines, growth factors and chemokines. ${ }^{18}$

The design of the present study obviously differed from those in previously reported investigations, and this must be taken into consideration while analyzing the data. Samples were not collected at standardized treatment points (e.g. at week 0,12 or 52) as in most previously reported studies, but during the patients' scheduled visits. While sample collection at standardized time points makes the data more homogeneous, it also involves certain methodical problems. As it would be unethical to continue the treatment of patients with a significant loss of response, these patients are often excluded from the analysis, and important data are therefore lost. Moreover, owing to their prospective nature, most of these investigations collect samples for only up to 52 weeks of treatment. The cross-sectional nature of the sample collection that we used, in contrast, allowed data collection even when the patients subsequently stopped the treatment due to the loss of efficacy. The sample homogeneity, of course, was not as high as in previous reports; however, as the patients appeared in random order for the appointments, the heterogeneity was balanced among the different analysis groups. Another advantage of our sample collection strategy was that the presence of ADA could be analyzed over a relatively long period of TNFi therapy (12-264 weeks). This allowed us to determine that antibody positivity is significantly more frequent among patients treated for less than 12 months. This observation suggests that the risk of ADA development is much higher during the first year of therapy. Consequently, the loss of therapeutic response after the first 12 months of TNFi therapy is likely to be caused by factors other than ADA. In these cases of late loss of efficacy, therefore, other possible factors (e.g. infection, nonadherence to treatment) should additionally be considered.

The main limitation of our cross-sectional approach is that patients recruited into this study might have represented a bias towards satisfactory clinical response to treatment. Patients with high antibody titers and consequent loss of response are likely to have a lower drug survival and are more likely to discontinue use of the drug. In contrast, patients using the biologic drug successfully for long periods of time are more likely to have undetectable levels of antibodies. By enrolling patients receiving treatment for at least 3 months, a positive selection bias might have been implemented.

Because of its ease of use, ELISA is the preferred method to measure the level of ADA. However, standard direct and indirect ELISA carry the disadvantage of producing non-specific binding and false-positive results. ${ }^{19}$ According to the published work, two assays stand out in terms of sensitivity and specificity: the bridging ELISA and the radioimmunoassay (RIA). RIA (and particularly its special format, the two-site assay) has very high specificity, and in addition, this method is less sensitive for drug interference than the bridging ELISA. Yet, a disadvantage is that the use of radioactivity hampers its broad application. Bridging ELISA reduce background readings by the requirement for two specific binding events for the target drug, which increases specificity of the assay. ${ }^{20}$

In conclusion, this study has provided evidence that the development of ADA during infliximab or adalimumab treatment of psoriasis is directly associated with reduced plasma TNFi concentration and increased plasma TNF- $\alpha$ level. A higher plasma TNF- $\alpha$ concentration may consequently lead to a wors- 
ening of the clinical symptoms of psoriasis. Clarification of whether the TNF- $\alpha$ concentration in the peripheral blood of patients receiving TNFi therapy is a direct indicator of the clinical activity of psoriasis demands further investigation. Furthermore, an increasing body of evidence supports the conclusion that monitoring the plasma drug level and the presence of ADA in daily clinical practice can aid therapeutic decisions during the TNFi treatment of plaque psoriasis.

ACKNOWLEDGMENTS: This work was supported by OTKA (Hungarian National Scientific and Research Fund) grant K73548 (R. G). The authors would like to thank Éva Viharosné Dósa-Rácz for the statistical analysis, Zsuzsanna Bata-Csörgö for a critical reading of the manuscript and Shannon Frances for proofreading the text.

CONFLICT OF INTEREST: Dr Kui and Dr Gaál were investigators and speakers for Abbott, Abbvie, Janssen-Cilag, MSD, Wyeth and Pfizer. Dr Gyulai and Dr Kemény were board members, investigators and speakers for Abbott, Abbvie, Janssen-Cilag, MSD, Galderma, Wyeth and Pfizer.

\section{REFERENCES}

1 Anderson PJ. Tumor necrosis factor inhibitors: clinical implications of their different immunogenicity profiles. Semin Arthritis Rheum 2005; 34: 19-22.

2 Rojas JR, Taylor RP, Cunningham MR et al. Formation, distribution, and elimination of infliximab and anti-infliximab immune complexes in cynomolgus monkeys. J Pharmacol Exp Ther 2005; 313: 578 585

3 Lecluse LL, Driessen RJ, Spuls PI et al. Extent and clinical consequences of antibody formation against adalimumab in patients with plaque psoriasis. Arch Dermatol 2010; 146: 127-132.

4 Takahashi $\mathrm{H}$, Tsuji $\mathrm{H}$, Ishida-Yamamoto $\mathrm{A}$ et al. Plasma trough levels of adalimumab and infliximab in terms of clinical efficacy during the treatment of psoriasis. J Dermatol 2013; 40: 39-42.

5 Tyring S, Gordon KB, Poulin Y et al. Long-term safety and efficacy of $50 \mathrm{mg}$ of etanercept twice weekly in patients with psoriasis. Arch Dermatol 2007; 143: 719-726.
6 Leonardi CL, Powers JL, Matheson RT et al. Etanercept as monotherapy in patients with psoriasis. N Engl J Med 2003; 349: 2014-2022.

7 Adisen E, Aral A, Aybay C et al. Anti-infliximab antibody status and its relation to clinical response in psoriatic patients: a pilot study. J Dermatol 2010; 37: 708-713.

8 Menter A, Feldman SR, Weinstein GD et al. A randomized comparison of continuous vs. intermittent infliximab maintenance regimens over 1 year in the treatment of moderate-to-severe plaque psoriasis. J Am Acad Dermatol 2007; 56: 31-44.

9 Menter A, Tyring SK, Gordon K et al. Adalimumab therapy for moderate to severe psoriasis: a randomized, controlled phase III trial. J Am Acad Dermatol 2008; 58: 106-115.

10 van Kuijk AW, de Groot M, Stapel SO et al. Relationship between the clinical response to adalimumab treatment and serum levels of adalimumab and anti-adalimumab antibodies in patients with psoriatic arthritis. Ann Rheum Dis 2010; 69: 624-625.

11 Mahil SK, Arkir Z, Richards G et al. Predicting treatment response in psoriasis using serum levels of adalimumab and etanercept: a single centre, cohort study. Br J Dermatol 2013; 169: 306-312.

12 Menting SP, van Lumig PP, de Vries AC et al. Extent and consequences of antibody formation against adalimumab in patients with psoriasis: one-year follow-up. JAMA Dermatol 2014; 150: 130-136.

13 Langley RG, Gordon KB. Duration of remission of biologic agents for chronic plaque psoriasis. J Drugs Dermatol 2007; 6: 1205-1212.

14 Reich K, Nestle FO, Papp K et al. Infliximab induction and maintenance therapy for moderate-to-severe psoriasis: a phase III, multicentre, double-blind trial. Lancet 2005; 366: 1367-1374.

15 Emi AN, de Carvalho JF, Artur Almeida SC et al. Immunogenicity of anti-TNF-alpha agents in autoimmune diseases. Clin Rev Allergy Immunol 2010; 38: 82-89.

16 Carrascosa JM. Immunogenicity in biologic therapy: implications for dermatology. Actas Dermosifiliogr 2013; 104: 471-479.

$17 \mathrm{Kim}$ J, Nadella P, Kim DJ et al. Histological stratification of thick and thin plaque psoriasis explores molecular phenotypes with clinical implications. PLoS One 2015; 10: e0132454.

18 Gottlieb AB, Chamian F, Masud $S$ et al. TNF inhibition rapidly down-regulates multiple proinflammatory pathways in psoriasis plaques. J Immunol 2005; 175: 2721-2729.

19 Mire-Sluis AR, Barrett YC, Devanarayan V et al. Recommendations for the design and optimization of immunoassays used in the detection of host antibodies against biotechnology products. $J$ Immunol Methods 2004; 289: 1-16.

20 Aarden L, Ruuls SR, Wolbink G. Immunogenicity of anti-tumor necrosis factor antibodies-toward improved methods of anti-antibody measurement. Curr Opin Immunol 2008; 20: 431-435. 